\title{
Remote Real-time Power Analysis and Management System
}

\author{
Syed Zain Rahat Hussain*: (iD), Minhaj Ahmed Moin*iD, Asad Osman*iD, Junaid Ahmed Memon**iD \\ *Department of Science and Engineering, Habib University - Karachi, Pakistan, 75290 \\ ** Department of Science and Engineering, Faculty of Science and Engineering, Habib University - Karachi, Pakistan, 75290 \\ (zain.rahat.hussain96@gmail.com,minhaj.moin@outlook.com,ao02772@alumni.habib.edu.pk,junaid.memon@sse.habib.edu.pk)
}

¥Corresponding Author; Syed Zain Rahat Hussain, Karachi, Pakistan, 75290, zain.rahat.hussain96@gmail.com

Received: 19.08.2021 Accepted:30.09.2021

\begin{abstract}
For many progressing nations, the electric energy measurement infrastructure is inadequate in terms of the lack of real-time measurement, as well as the connectivity to the central data collection station. To overcome such issues, more modern methods of sensing and distributing electrical energy must be explored and implemented. In this article, we present a costeffective LoRa (Long Range) based real-time load monitoring and control system that can measure the amount (voltage, current, and power) and the quality (power factor and harmonics) of the electricity being supplied using a microcontroller-based Data Acquisition System, and can also gather and present that data, as well as provide remote power cut and restore functionality through an Internet-of-Things SCADA (Supervisory Control and Data Acquisition) server. The purpose of this study was to demonstrate the benefits of real-time energy measurement, and how such a system can help tackle Pakistan's energy crisis. The system is powered by the power line, and by a battery backup system to prevent data loss in the event of an outage/fault. Through software simulations, the system was evaluated before being implemented on the hardware, and results of both are described. The system was energy efficient, owing to the usage of the LoRa wireless technology and low power consuming components, drawing only $680 \mathrm{~mA}$, and was accurate in analyzing the power consumption characteristics of loads in real-time, tested in comparison to standard laboratory equipment, where the error of the Data Acquisition System was found to be around $0.9 \%$, $2.91 \%$, and $1.6 \%$ for the voltage, current, and frequency respectively.
\end{abstract}

Keywords Pakistan, Data Acquisition System, LoRa Wireless Communication, SCADA Server, Internet-of-Things.

\section{Introduction}

Many developing nations are unable to meet their burgeoning energy demands in the twenty-first century, due to deficiencies in their power distribution infrastructure and persisting challenges such as theft, waste, and inefficiency. One of these countries is Pakistan, where power outages caused by load shedding and breakdowns interrupt people's lives and cause innumerable damage, as electricity is the lifeblood of the technology that is relied so much on. In 2012, consumers were without electricity for an average of 1453 hours due to outages that occurred around five times per day [1]. For the utilities, issues that continue to plague them and cause them to lose money are theft and meter tampering, where people get access to electricity without paying for it. The number of power nonpayers increased to 5.3 million in Pakistan during the year 2017-2018, with the total theft amount adding up to Rs 404.8 billion [2].

In Pakistan, analog meters were expected to account for about half of all meters used in 2016 [3]. Digital meters have grown more widely since then, now being used for both home and business metering purposes. These have certain benefits, such as increased efficiency and anti-tampering features, however, cannot send their readings at regular intervals to a utility-controlled database and must be read manually, as opposed to the advanced, but less prevalent and costlier Automatic Meter Reading (AMR) and Smart Meters [4]. There has been opposition to Smart Meters in Pakistan in the past since customers believed that these meters ran faster and energy firms were billing them unfairly [4]. To remedy this, providing customers with the necessary information might help dispel misunderstandings and promote the adoption of this new technology.

According to the paper by G. Barai, S. Krishnan, B. Venkatesh, Advanced Metering Infrastructure (AMI) as a real-time load monitoring system can efficiently manage the power supply, and optimize resource allocation and usage [5], which reduces the load on the Grid, as well as operational costs. AMI can also help in detecting electricity theft, thus saving revenue [6]. It can remotely switch on or off a 
particular customer's supply if it detects a load higher than the sanctioned load for that particular customer type. Once the theft is detected a notification is provided to the electricity utility server to take the necessary action. The AMI system proposed in [6] is designed to send an SMS to the electricity supplier server upon detecting an unauthorized load siphoning power from the grid.

In [7], the authors propose a system applicable to the Indian Electric Supply Standard, similar to the Pakistan standard, which is $230 \mathrm{~V}$ Single Phase, $415 \mathrm{~V}$ Three Phase, and $50 \mathrm{~Hz}$. The design in this paper includes demand-side load management and load limiting. By continuous monitoring of the power data from the customers, the smart grid can automatically provide a load profile for a particular area or an individual customer, so the electric distributor can plan their supply accordingly. Upon using up more power than allowed, a customer's supply can be stopped, until they lower their load and manually switch their power back on, with each of these events being communicated back to the supplier [7].

AMI can also be integrated with the modern Internet-ofThings [8]. The smart meter can be configured to measure the consumption of the customer's electricity at desired frequencies, which can then be uploaded to the remote utility server, eliminating the need for manual meter readings, which can sometimes be erroneous, leading to incorrect billing. Connecting the meter to the Internet also allows the customers to view their consumptions on an online portal or a smartphone app with location markers as well [9]. This would lead to a more conscious use of energy as a result of the increased awareness.

A Smart Grid also allows the easier integration of renewable energy sources, reducing the need to burn harmful fossil fuels [10], and also promotes consumer integration through Net Metering and Distributed Generation. With Distributed Generation, consumers are able to produce their energy through various renewable energy sources, and also store them in batteries or storage devices, or provide that energy to the Grid, reducing the cost of their total electricity bill [10].

However, certain considerations must be made in the implementation of AMI, as discussed in [11]. These include challenges related to the data security of the smart grid. As the AMI Smart Grid is itself a big source of big data generation, there are many data management concerns related to it and multiple potential weak points in its structure from a cyber and networks security aspect [12]. Each meter connected to it is a port to the whole system, which means if one meter is compromised, the whole system could be brought down. Due to this, it is required to have a powerful enough server that can handle large amounts of data, while also ensuring high levels of data encryption and security. The System design also has to be scalable to a larger area and network while ensuring maximum availability and minimized downtime during maintenance [11].

The goal of this paper was to showcase an architecture for real-time distribution power monitoring that was both cost-effective and efficient. The technology was meant to highlight the advantages of real-time energy measurement and how it might aid in alleviating Pakistan's energy shortage situation. With the proposed system, electrical characteristics of the power being supplied, such as single-phase voltage, current, power, frequency, power factor, and harmonics were measured in real time, along with additional features such as remote switching, GPS synchronization, and a backup power supply. The system used LoRa technology to achieve bidirectional communication, with the measured data being transceived with an IoT SCADA server through a Raspberry Pi-based gateway. The data measurement system was intended to be cost-effective, and was also simulated to ensure that its design would work effectively. To verify its accuracy, the data acquisition system was calibrated with a standard lab digital multimeter (Gwinstek GDM-8245), and an oscilloscope (Gwinstek GDS-2000A).

A similar system was proposed in [13], and was deployed as a pilot project in Bali, Indonesia as part of the Bali Eco Smart Grid. 1000 bi-directional smart meters were tested and the results were that the new smart grid system gave high satisfaction to the consumers, and high success in the meter reading rate, accuracy, efficiency, control, and reduced operational costs to $90 \%$ of the pre-existing system. In the end, it was recommended that such smart meters be deployed to all customers in Bali the region [13].

\section{System Architecture}

The system consisted of four modules, as shown in Fig. 1. Each component's role and design details are provided in the next section.

Data Acquisition, Control, and Processing Unit: This module was responsible for sensing the voltage and current signals, conditioning them to the required amplitude range, and processing them. The voltage and current sensing were carried out by a ZMPT101B AC voltage sensor and an ACS712 5A current sensor respectively. The requirement for accurate voltage measurement as a range was from $180 \mathrm{~V}$ to $250 \mathrm{~V}$ per phase. The ZMPT101B voltage sensor consisted of a transformer to step down the mains voltage connected to it, to a $2.5 \mathrm{~V}$ peak signal, and then with an additional offset of $2.5 \mathrm{~V}$, to make the final output signal range from $0 \mathrm{~V}$ to $5 \mathrm{~V}$, which could be read by the microcontrollers analog-to-digital converter (ADC). The ACS712 had a hall-effect sensor to convert the current signal to a 0 to $5 \mathrm{~V}$ voltage signal, depending on the magnitude of the current. A zero-crossing detector circuit was used for voltage frequency measurement and phase detection, while a GPS module (NEO-6M) provided location and time-date synchronization. These sensors were connected to an Arduino MEGA 2560 microcontroller, which was responsible for the signal processing, and for transmitting the data to the Gateway. A relay was connected to remotely switch $\mathrm{OFF} / \mathrm{ON}$ the power to the mains if needed. A battery backup unit was also incorporated to provide electricity to the different parts of the system, both during regular working and in the event of a power loss. 
LoRa Communication Radios: The Internet Gateway and Data Acquisition Unit were both connected to LoRa Radios for wireless communication between them. LoRa was selected for this, as it was found to be the most cost effective and had low overall power consumption, while also giving very high ranges by using very low frequency/low bandwidth signals. To allow the data to be sent over long distances, the data size had to be kept as small as possible, and for that, instead of transmitting it in its raw state, the data was first processed and sampled before being transmitted. Since the data used for metering was in the range of a few $\mathrm{kBs}$, LoRa was a viable option for such purposes. For the Meter/Data Acquisition System, the LoRa Radio used was the SX1276 based Dragino LoRa Shield, and for the Internet Gateway, the RF96 based Dragino LoRa GPS Shield was used.

Internet Gateway: The Gateway was responsible for maintaining the connection to the Internet, and managing the data being transmitted to and from the LoRa Radio network. It consisted of a Raspberry $\mathrm{Pi}$ and an Arduino UNO Microcontroller connected to the LoRa Radio. While the Pi was connected to the Internet and uploaded the received data to the SCADA Server once every two seconds, the Arduino UNO handled all communication between the $\mathrm{Pi}$ and the LoRa Radio.

SCADA Server: The SCADA Server was used for remotely managing and monitoring the parameters of the system, with the data coming from the Gateway through an MQTT protocol, which is a low bandwidth messaging protocol for IoT applications. Ignition SCADA was used as the SCADA Software for the system. A Human Machine Interface (HMI) showed the information coming in from the connected meters. It could also generate alarms, scheduled reports, remotely set power consumption limits for a certain meter, remotely shut off power to any connected meter, and could export the measured data to a CSV file for use in data analysis software. It could be accessed from any device connected to the Internet within the network, and with the authorized credentials.

\section{Design Challenges}

The common mains power supply in Pakistan is rated at 220-240 VRMS Single Phase, and 400V Three Phase, at $50 \mathrm{~Hz}$ Frequency. Normally, residential consumers have a current usage of around 12 to 20 Amperes, but can go up to as much as 30A. During normal operation, the mains voltage can go down as low as $180 \mathrm{~V}$ in certain cases. The meter, once properly calibrated, can measure these lower voltage values as well, however, due to noise, the output is prone to errors.

Considering the $50 \mathrm{~Hz}$ Frequency of the power being supplied, the sampling rate of the system, according to Nyquist Theorem, would need to be at least $100 \mathrm{~Hz}$. An ideal sampling rate is 5 to 10 times the base frequency. Keeping this in mind, the meter was designed around a sampling rate of around $1000 \mathrm{~Hz}$, which is sufficient for accurate sampling.

For the prototype, the sensor that was used had a current rating of $5 \mathrm{~A}$. The current rating of the actual meter can be made to be up to $30 \mathrm{~A}$. The $30 \mathrm{~A}$ sensor was not used for the prototype, as the lower currents of $1 \mathrm{~A}$ to $2 \mathrm{~A}$ would have had a higher error. The $30 \mathrm{~A}$ sensor would map $-30 \mathrm{~A}$ peak to $0 \mathrm{~V}$ and $30 \mathrm{~A}$ peak to $5 \mathrm{~V}$, being much less sensitive to smaller currents.

\section{A. Using the ZMPT101B for Voltage Measurement}

The voltage sensor module had to be calibrated before use, and this was accomplished by taking the reading from the sensor with the mains voltage first switched OFF. The purpose of this was for removing any pre-existing bias in the readings. A multiplication factor was then used to scale the readings from the voltage sensor to the values measured by the multimeter with the mains voltage turned ON. One issue
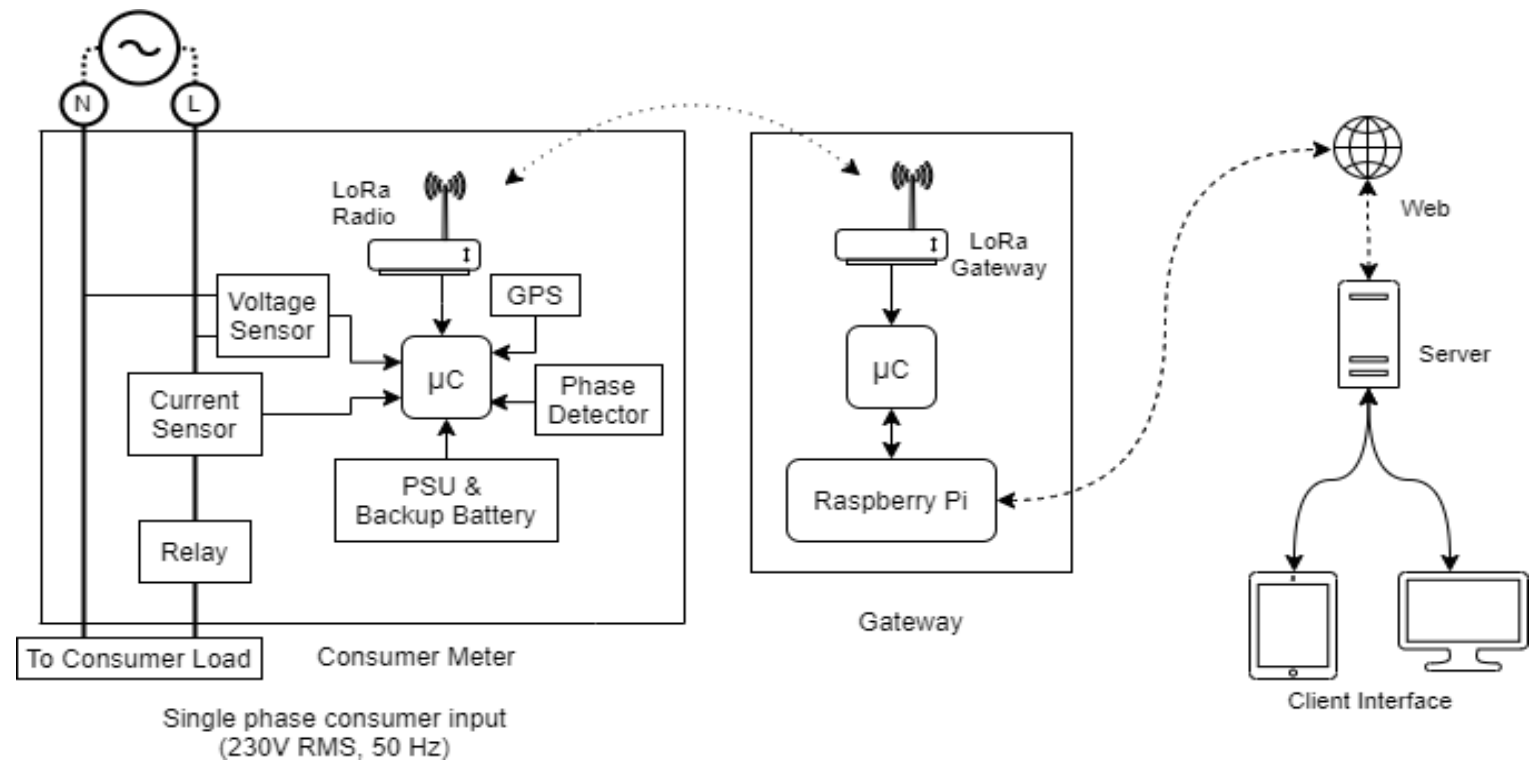

Fig. 1. System Architecture showing the Data Acquisition System, LoRa Wireless Communication, Internet Gateway, and SCADA Server 
encountered with the ZMPT101B voltage sensor was that while it was supposed to output $5 \mathrm{~V}$ peak according to its datasheet, in testing, the output signal started to saturate around $3.5 \mathrm{~V}$, with the signal getting lost beyond that. For this, the gain of the voltage sensor was adjusted to about $3.6 \mathrm{~V}$ peak. This problem occurred due to the LM358 OperationalAmplifier IC used in the ZMPT101B. The LM358 had a max output of $\mathrm{VCC}-1.5 \mathrm{~V}$, with the bias being added as $\mathrm{VCC} / 2$. As a result, the total range of the sensor was reduced to $1.4 \mathrm{~V}$ to $3.6 \mathrm{~V}$, instead of $0 \mathrm{~V}$ to $5 \mathrm{~V}$, lowering the resolution and accuracy of the sensor.

\section{B. Meter Power Supply and Battery Backup System}

The metering system required a constant DC voltage supply to function, even in the absence of electricity such as during a malfunction, to carry out fault diagnosis. As a result, there was a need for a separate power source as backup for those scenarios. To accommodate this, the DC power supply for the meter included the functionality to provide power from two parallel connected Li-Ion cells, as well as the mains power. The Li-Ion cells could only provide $3.7 \mathrm{~V}$, so an MT3608 boost converter was used to step it up to $5 \mathrm{~V}$. The batteries were charged when the mains power was working and connected, through a TP4056 charging module. This was used to power every part of the Data Acquisition unit, including the metering system and the LoRa radio. The architecture of the meter power supply and battery backup system is visible in Fig. 2.

\section{Frequency Measurement and Harmonics}

The frequency was determined via a hardware based zerocrossing detector due to the high processing and memory requirements of FFT (Fast Fourier Transform), and the memory and computational restrictions of the microcontroller

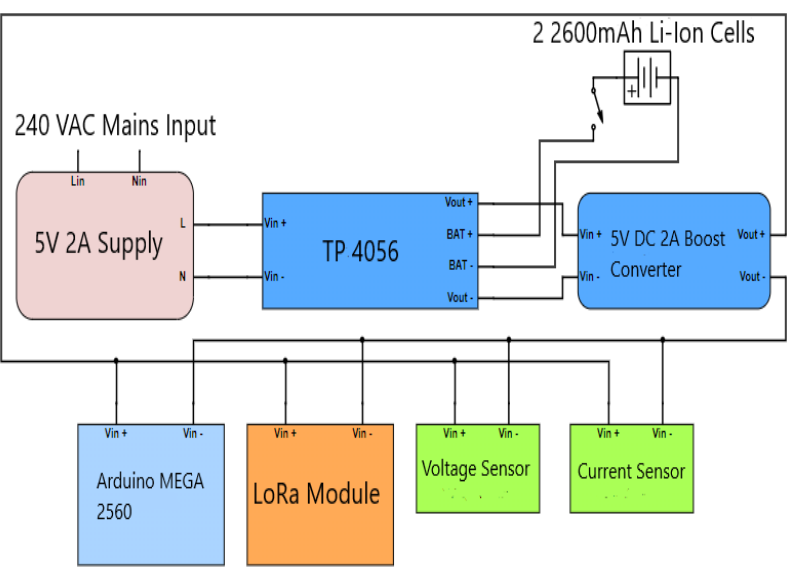

Fig. 2. Architecture of the Power Supply and Battery Backup Unit

utilized. Instead, the FFT was only used to measure relative amplitudes of the current harmonics. The zero-crossing detector converted the sine wave output from the ZMPT101B voltage sensor into a square wave signal, which was then input to an interrupt pin of the microcontroller. Using this interrupt pin, an internal timer of the microcontroller was started and stopped, to get the pulse timings for the HIGH and LOW pulses. The pulse timings were then added together, and then inverted to get the fundamental power frequency.

\section{Design Validation}

\section{A. Proteus Simulation of the Data Acquisition Module}

A software simulation of the Data Acquisition System, including the onboard sensors (the ZMPT101B and ACS712 5A), signal conditioning circuitry, and the microcontroller

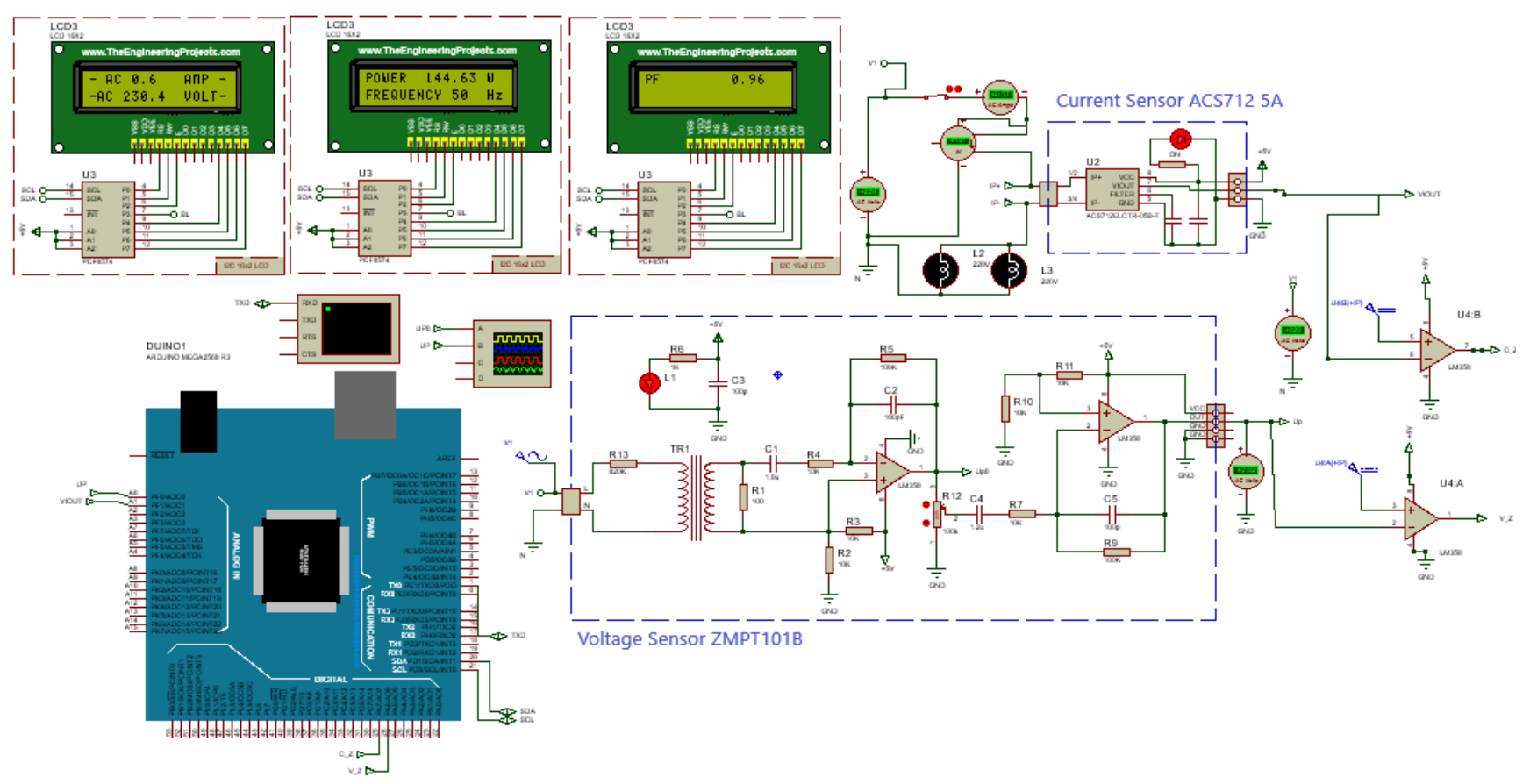

Fig. 3. Proteus simulation of the Data Acquisition module showing voltage and current sensors 
were done on Proteus 8.9 Software, visible in Fig. 3. For the testing, the mains voltage to be measured was set to $230 \mathrm{~V}$ RMS, two $60 \mathrm{~W}$ bulbs were connected in parallel to act as the load, and the virtual microcontroller was programmed with the same code as the actual one used in the hardware. Output of the microcontroller was shown on a virtual $16 \times 2$ LCD Module.

\section{B. Theft Detection Simulation using Proteus Software}

To demonstrate the electric theft detection function of the system, two sets of the meters were simulated, one being a distribution side meter (on the left in Fig. 4), and the other being a consumer side meter (on the right in Fig. 4). In normal circumstances, the sum of all currents being measured by the consumer side meters would be equal to the current reading of the distribution side meter. However, if there was higher current being measured at the distribution side, then there was an additional electrical load not accounted for, and would indicate theft taking place. In Fig. 4, it was shown through an ammeter, that without any theft load connected, the current readings at both the consumer and distribution side meters was the same. However, when the theft load was connected to the circuit, the current value at the distribution side was higher, indicating the theft.

\section{Power Supply Simulation on MATLAB Simulink}

To calculate the max current draw of the system, the individual and total current draw of the system is shown in the Table 1, which added up to $680 \mathrm{~mA}$. The backup power supply was made with twice this consumption, in the case that more equipment was added later, so that all the components would function properly. Hence, the battery backup supply system was designed around $1360 \mathrm{~mA}$ of peak consumption
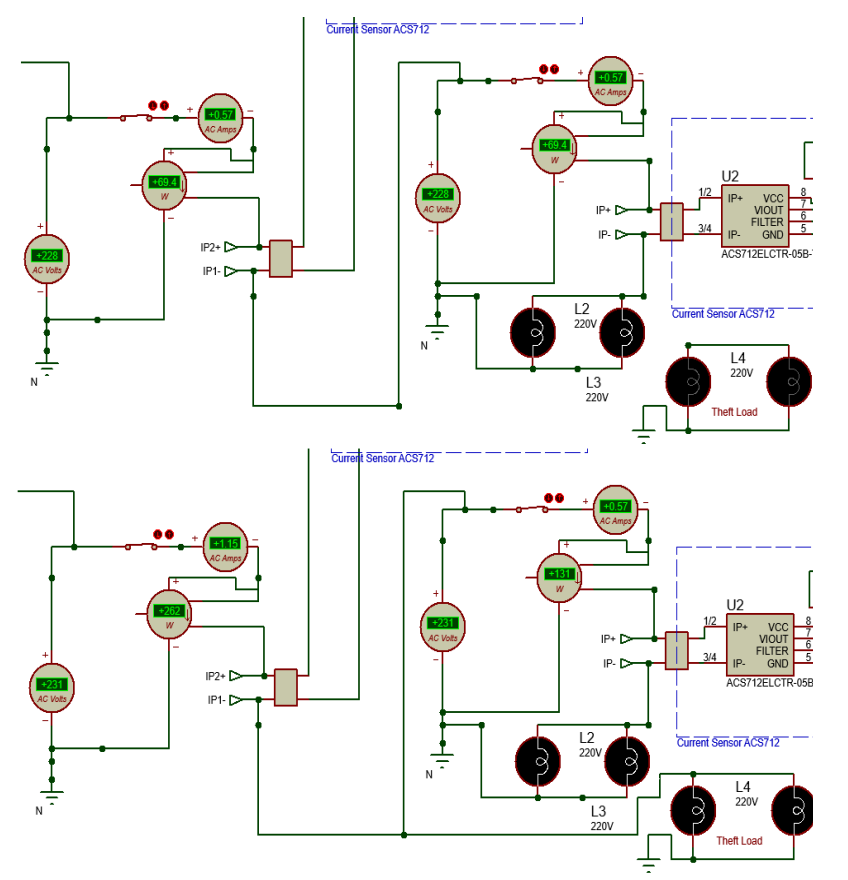

Fig. 4. Proteus simulation for theft detection
Table 1. Current draw for each component of the Metering System

\begin{tabular}{|l|l|}
\hline \multicolumn{1}{|c|}{ Component } & Current Consumption \\
\hline Arduino Mega & $500 \mathrm{~mA}$ \\
\hline ZMPT101B & $10 \mathrm{~mA}$ \\
\hline ACS712 & $10 \mathrm{~mA}$ \\
\hline LoRa Shield & $160 \mathrm{~mA}$ \\
\hline Total & $680 \mathrm{~mA}$ \\
\hline
\end{tabular}

of the system. The battery of the backup system consisted of two Li-Ion cells connected in parallel, with a total of $5700 \mathrm{mAh}$ capacity, and a $\mathrm{C}$ rating within the range 0.2 and 1C. Using this information, the charging and discharging times of the battery were found. The discharge time was found as 4.2 hours, and the optimal $\mathrm{C}$ rating was 0.239 . The simulation of the power supply was also done in MATLAB, shown in Fig. 5a. Resistors were used to model the different parts of the Data Acquisition module, including the Arduino MEGA 2560, LoRa Shield, and current and voltage sensors. For any additional loads, a fourth resistor was added to model any extra loads. As is visible from the graphs in Fig. 5a, the battery charge decreased linearly from $80 \%$ to $0 \%$, with no major change in the voltage and current output of the power supply, which remained at $4 \mathrm{~V}$ and $1.4 \mathrm{~A}$ respectively (the current was previously calculated as $1360 \mathrm{~mA}$ ). The cursors in the graph, visible in Fig. 5b and 5c, showed the discharge time to be almost 15000 seconds, or roughly 4.16 hours, which was very close to the calculated value of 4.2 hours.

\section{Hardware Prototyping}

The hardware for the End Node making up the Data Acquisition module, along with the Internet gateway (the Internet router and Raspberry Pi) are visible in Fig. 6a and Fig. 6b respectively, while Fig. 7 shows the power supply unit. All of the tested loads were connected to the

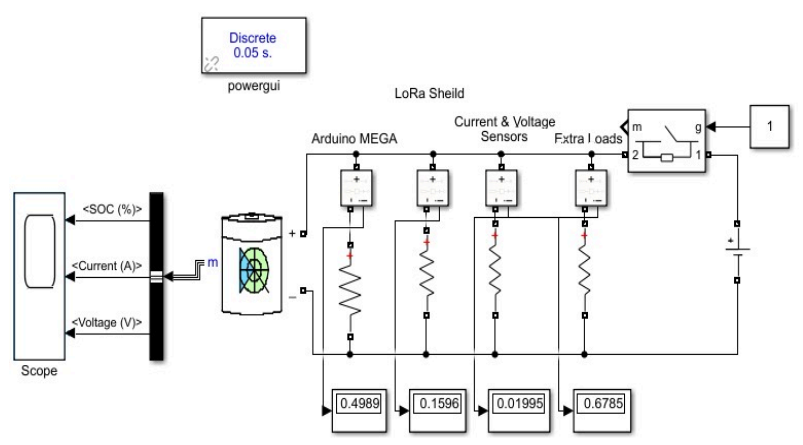

Fig. 5a. MATLAB Simulink Simulation of the Power Supply 

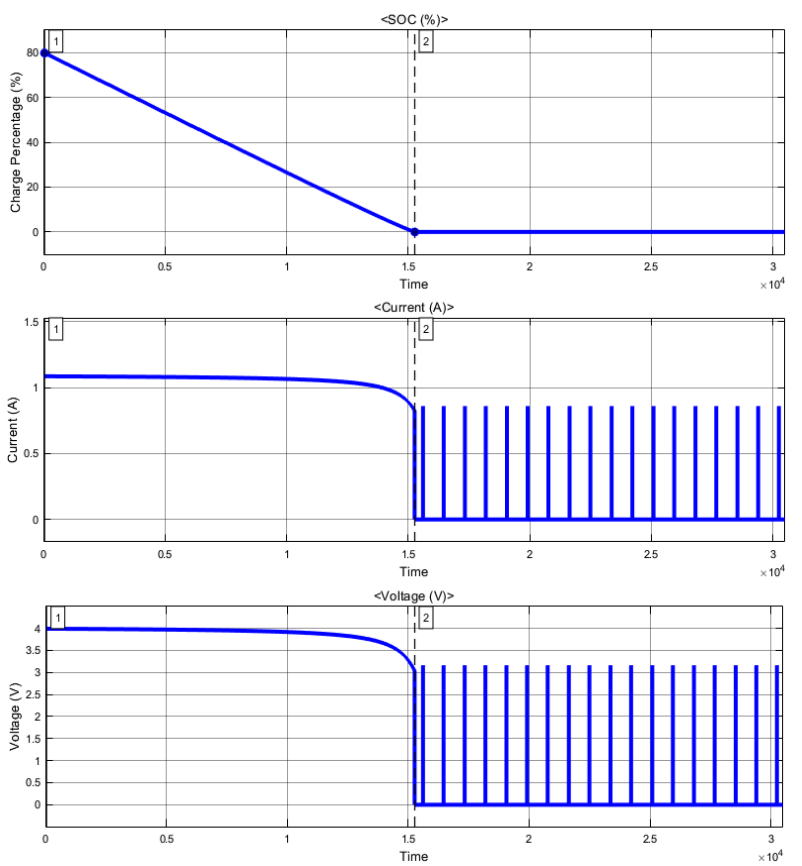

Fig. 5b. Graphs of Voltage and Current in MATLAB; Discharging Mode

switchboard, which was connected to the mains supply along with the sensors and the relay.

\section{A. Voltage Measurement}

The gain setting of the voltage sensor was calibrated and its value was adjusted through a potentiometer on the voltage sensor to map a $215 \mathrm{~V}$ input to $2.65 \mathrm{~V}$ sensor output. This reference voltage value was used for converting and scaling the ADC output values back to the actual voltage. A UNI-T UT-51 multimeter was used to check and compare the actual

voltage values to the scaled values from the Data Acquisition module. Fig. 8 shows the voltage value measured by the Data Acquisition module shown on the HMI, being compared to multimeter output. The multimeter voltage reading and sensor reading differed by $2 \mathrm{~V}$ at most, which meant an error of $0.9 \%$.

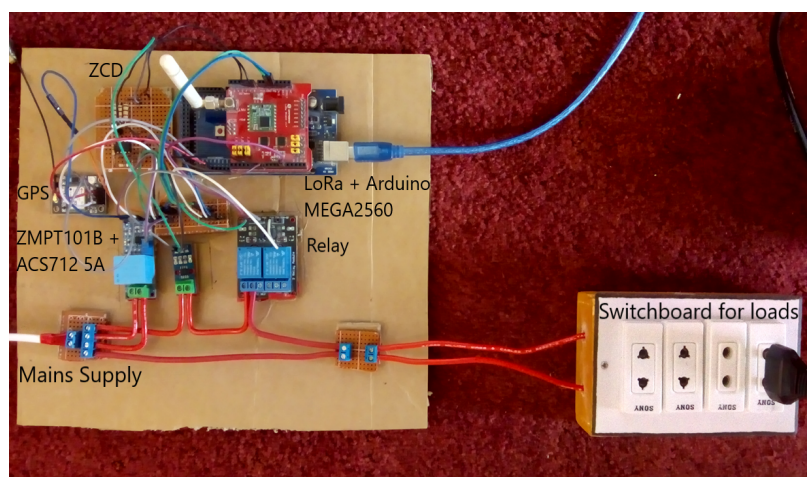

Fig. 6a. End Node Hardware Implementation

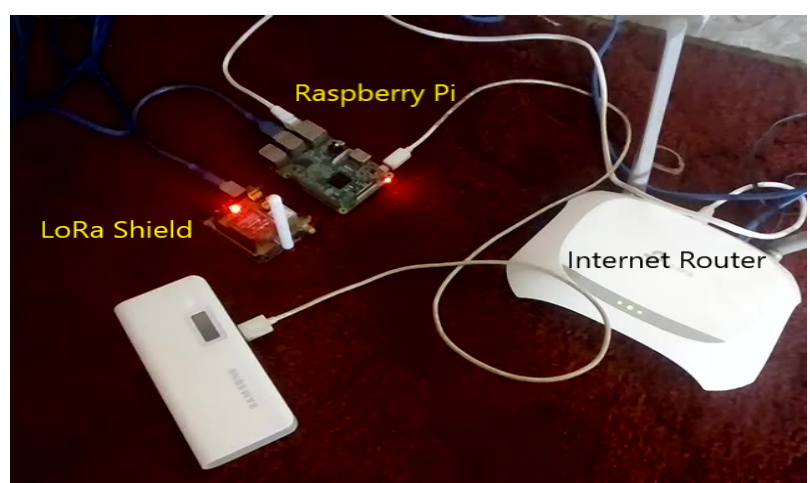

Fig. 6b. Internet Gateway Hardware Implementation

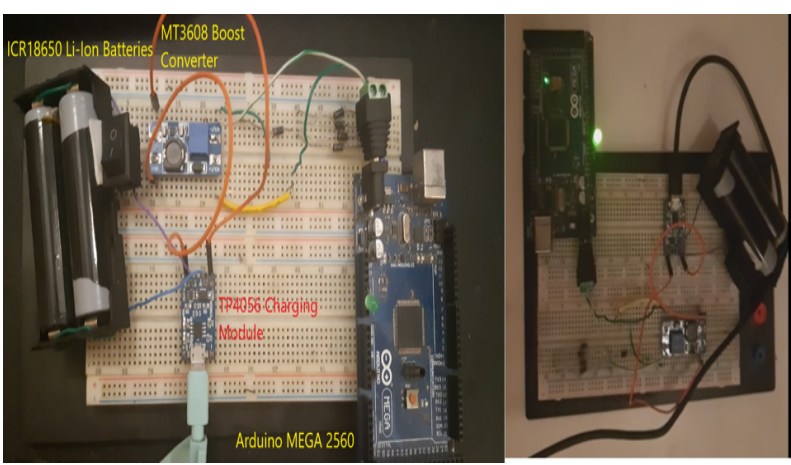

Fig. 7. Battery backup and Power Supply Unit Hardware Implementation

\begin{tabular}{|c|c|c|c|}
\hline \multicolumn{2}{|r|}{ Time } & \multirow{2}{*}{\multicolumn{2}{|c|}{$\begin{array}{c}\text { Value } \\
7.976 \mathrm{e}+01\end{array}$}} \\
\hline 1। & 44.203 & & \\
\hline $2 !$ & 15270.279 & & $0.000 \mathrm{e}+00$ \\
\hline \multirow[t]{3}{*}{$\Delta \mathrm{T}$} & $15.226 \mathrm{ks}$ & $\Delta \mathrm{Y}$ & $7.976 \mathrm{e}+01$ \\
\hline & $1 / \Delta \mathrm{T}$ & & $65.677 \mu \mathrm{Hz}$ \\
\hline & $\Delta \mathrm{Y} / \Delta \mathrm{T}$ & & $5.239(/ \mathrm{ks})$ \\
\hline
\end{tabular}

Fig. 5c. Cursor Information for the MATLAB Simulation

\section{B. Load Test}

Load Tests were performed to check the current waveforms of the different loads, and the plots were made with the raw, uncalibrated sensor outputs. The load tests were performed on a Switch Mode Power Supply (non-linear

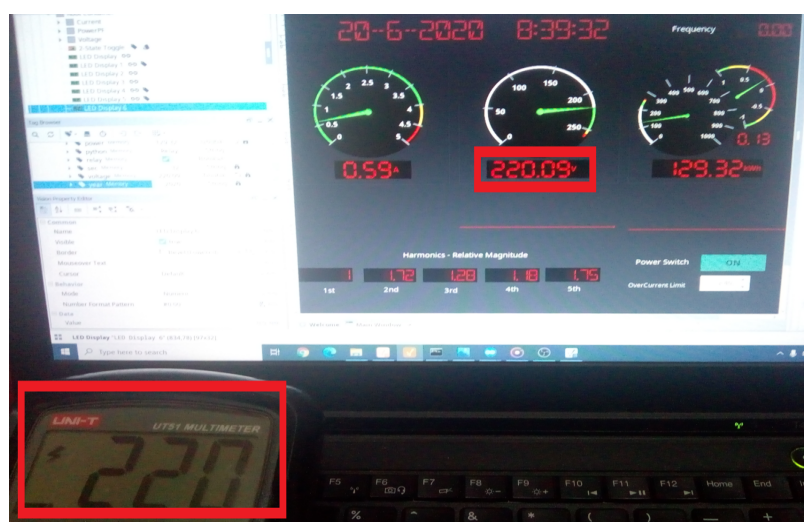

Fig. 8 Data Acquisition System voltage measurement shown on HMI vs direct multimeter output. 

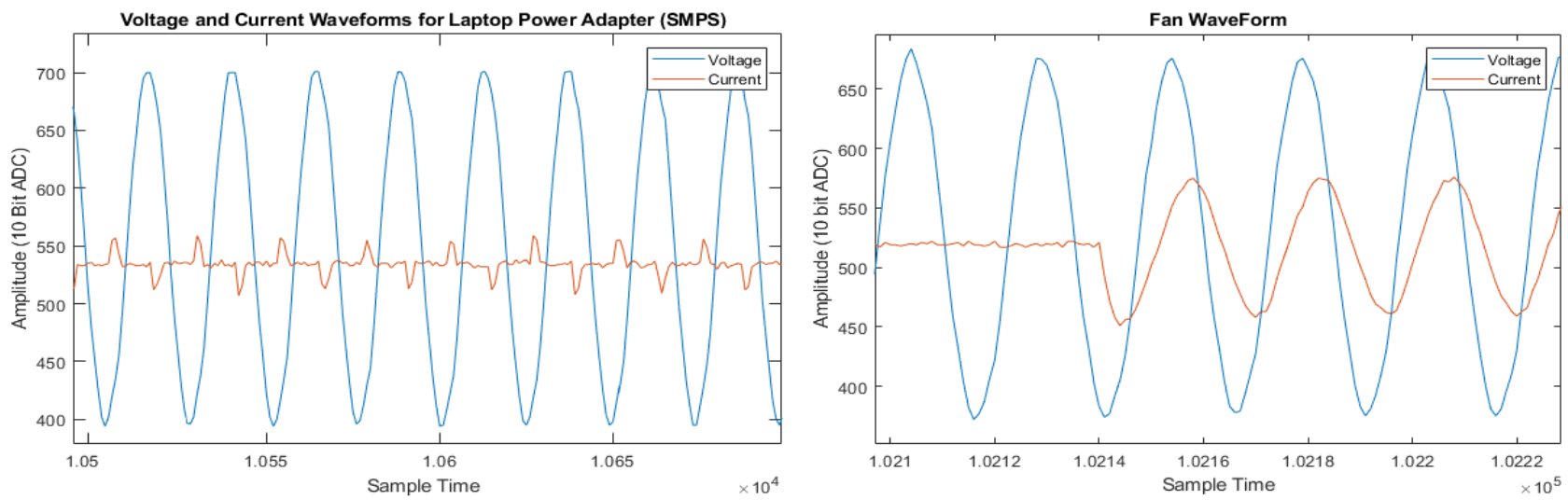

Fig. 9. Laptop charger + Fan waveforms measured by Data Acquisition System

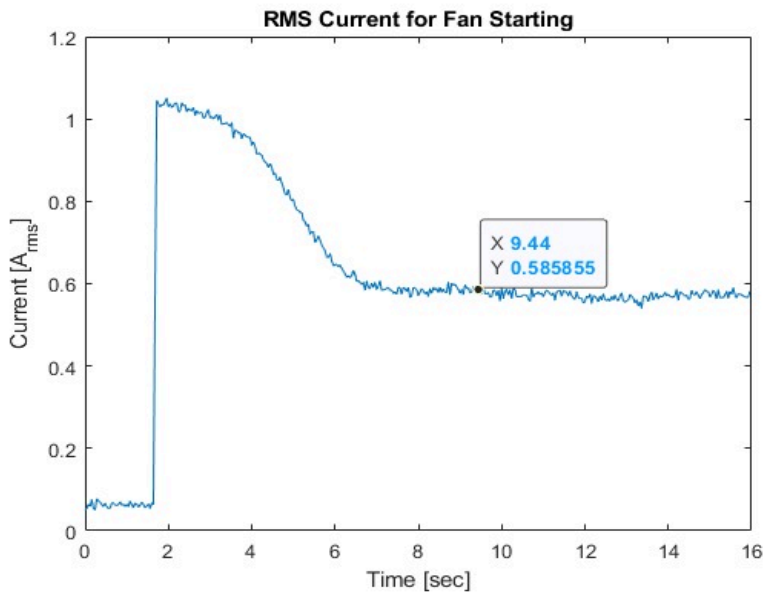

Fig. 10. RMS current values for the fan when it is OFF, when it is starting, and when it is at steady speed

load), and a standing fan (inductive load). The waveforms for both loads are shown in Fig. 9. The fan did not produce harmonics, but the current waveform lagged behind the voltage waveform, as expected of an inductive load. The current waveform for the fan also showed a higher starting current that slowly tapered off to its steady state value, caused by the high starting torque of the fan motor, highlighted in Fig. 10. As expected, the TRIAC in the Switch Mode Supply allowed only a portion of the sine wave, producing odd harmonics in the signal.

The Inductive Load (Fan) had a steady state current draw of around 0.58A RMS, as measured by the digital multimeter visible in Fig. 11. At the same time, the HMI showed 0.6A RMS current consumption for that load (as was seen in Fig. 8 ). The percentage error for the current measurement can be calculated as $(0.6-0.583) / 0.583=2.91 \%$. Since the ACS712 current sensor is manufactured for use with heavier loads, it would be more accurate for higher currents, reducing the error further.

Due to this slight nonlinearity in the sensor, the current also shows up as $0.1 \mathrm{~A}$ or $0.2 \mathrm{~A}$ when there is no load connected. This has to do with the resolution of the current measurement. In the hardware experiments, the $5 \mathrm{~A}$ current

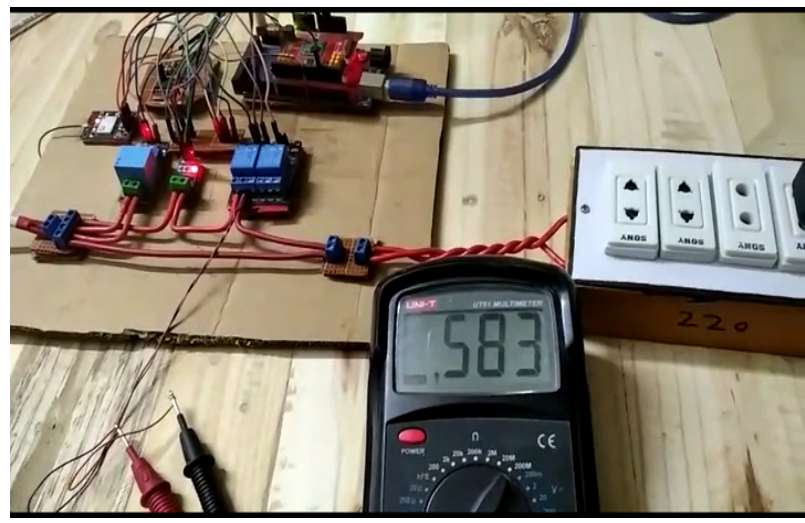

Fig. 11. Digital Multimeter Reading of the Fan current draw during normal operation

sensor was used, which maps to the 0-5V Range of the ADC. As a result, a $-5 \mathrm{~A}$ Peak would map to $0 \mathrm{~V}$ and $5 \mathrm{~A}$ peak would map to $5 \mathrm{~V}$. In the case of no current, there would a $2.5 \mathrm{~V}$ output to the ADC. Due to noise, if that value were to be $2.6 \mathrm{~V}$. it would show as 0.1-0.2A RMS, visible in Fig. 12. So at lower currents, the error percentage is going to be higher.

\section{Frequency Measurement}

The zero-crossing detector took in the signal from the voltage sensor as input and converted it into a square wave,

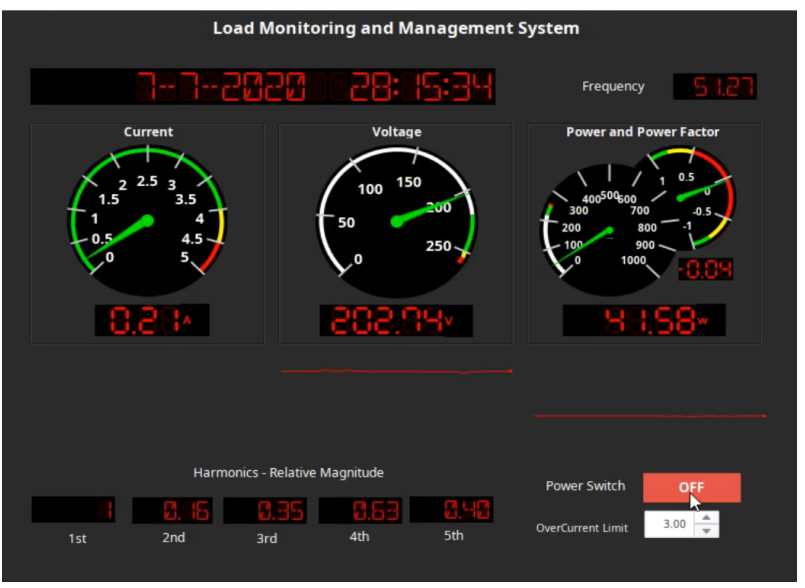

Fig. 12. HMI with the Supply switched OFF via the remote-control 


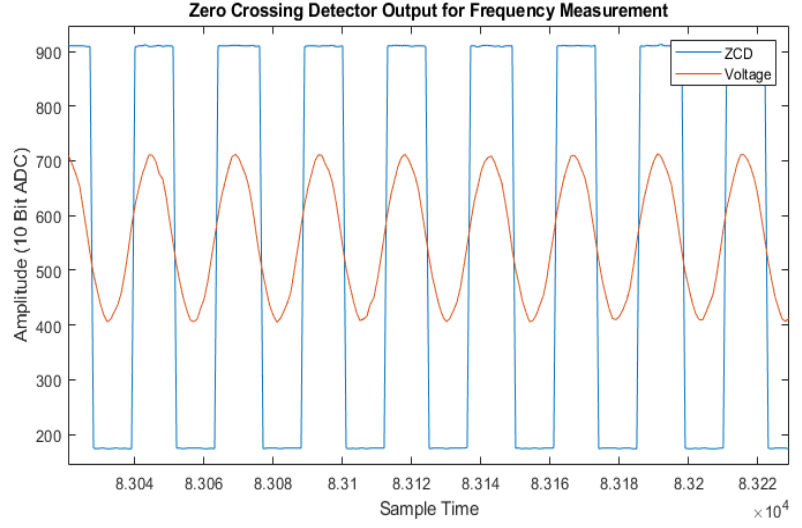

Fig. 13. Output of the Zero-Crossing Detector for frequency measurement

which served as a digital input to the Microcontroller, shown in Fig. 13. The square wave was used to drive a digital interrupt that started and stopped an internal timer in the microcontroller, and thus, gave the times for the HIGH and LOW pulses. These times were added, and then inverted to get the frequency. The frequency calculated through this approach was found to be close to 50 to $50.8 \mathrm{~Hz}$, resulting in an error of at most $1.6 \%$. For measuring the harmonic frequencies in the current waveform, an FFT Algorithm was also used with the SMPS as the load, a visualization of which is shown in Fig. 14. Due to the relatively slow sampling rate and memory of the Arduino MEGA 2560 (1500 Samples Per Second and 8KB SRAM), the accuracy of the FFT Algorithm was not very high, and the peaks for the subsequent harmonics seemed to deviate towards higher frequencies, which was why the HMI only showed the harmonics up to the 5 th $(250 \mathrm{~Hz})$. The relative magnitudes of the haromincs measurement were visible on the HMI numerically, visible in Fig. 16.

\section{Power Factor Measurement}

The power factor of the load is calculated by first passing the output of the voltage and current sensors through a low

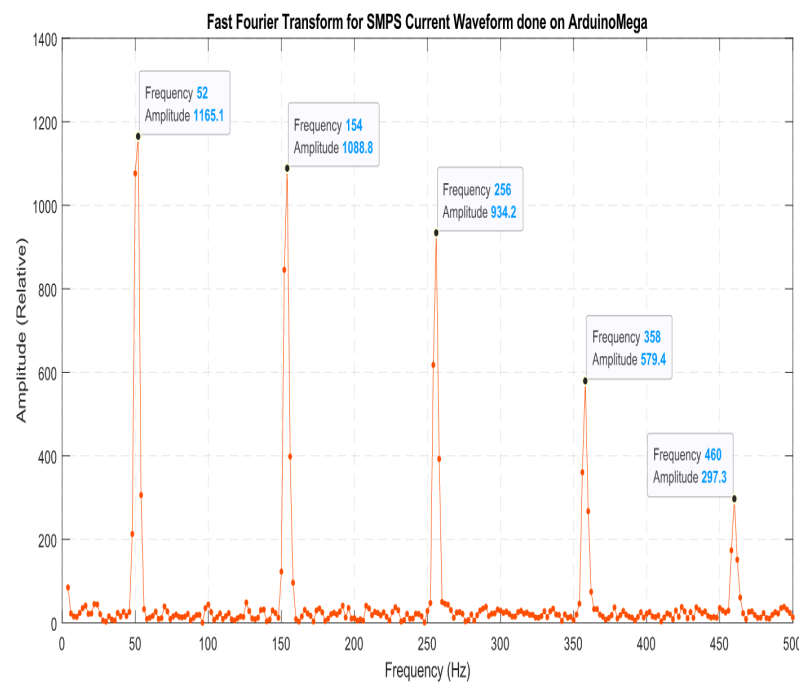

Fig. 14. Output of the FFT run on the SMPS Current Waveform

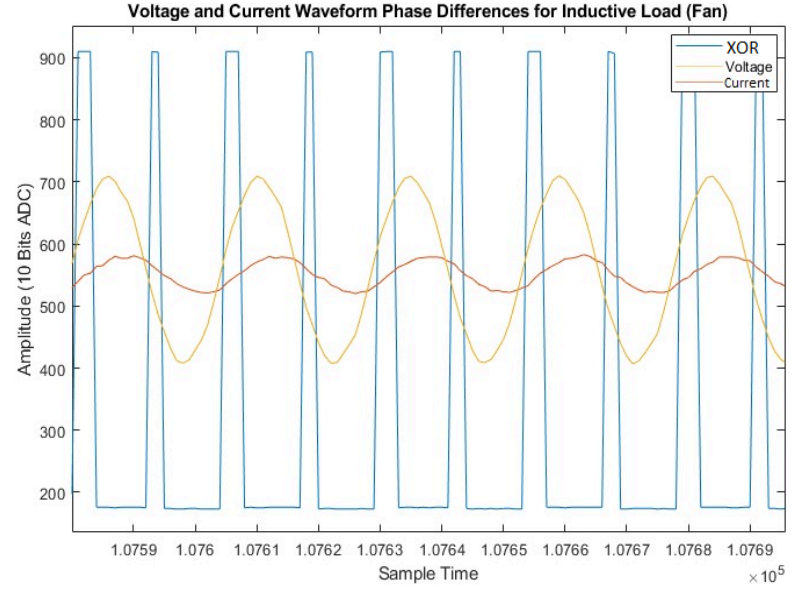

Fig. 15. XOR gate output for Power Factor Measurement

pass filter that extracts the fundamental frequency of $50 \mathrm{~Hz}$, and then to a zero-crossing detector that converts the sine wave signal into a square wave. The two square waves of the current and voltage signals are then input to a XOR gate that gives a HIGH output when the square waves are not equal in time, shown in Fig. 15. The phase lag from reactive loads will cause one signal to lag and one to lead, so the output of the XOR gate will be HIGH for the phase lag time of the waves. That time can then be measured by a timer in the microcontroller triggered by the edge outputs of the XOR gate, giving the time difference. From the time difference the phase angle can be calculated as:

Phase Angle $=360 \times($ time difference $\div$ time period $)$

The cosine of the calculated phase angle is going to give the Power Factor. For inductive loads, the power factor would be somewhere around 0.8, and for Switch-Mode Power Supplies, the PF would be low, around 0.3 to 0.2 . This was in line for what was measured by the Data Acquisition and displayed on the HMI.

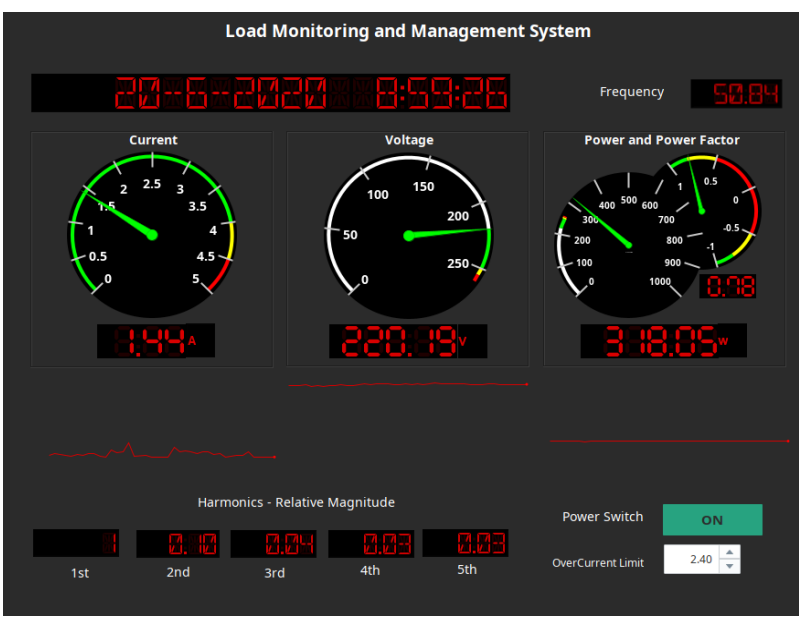

Fig. 16. HMI on the SCADA server displaying measurements from the End Node 


\section{E. Internet Gateway, SCADA Server, and HMI}

The Internet Gateway received the power quality data and statistics over the LoRa Network, parsed the data into the correct format, and sent it over to the SCADA server to be saved into the database via a Machine-to-Machine Messaging Protocol for IoT Devices (MQTT), that greatly reduced the time it would take to complete. Once the SCADA received the data, it updated the HMI to reflect the change. The

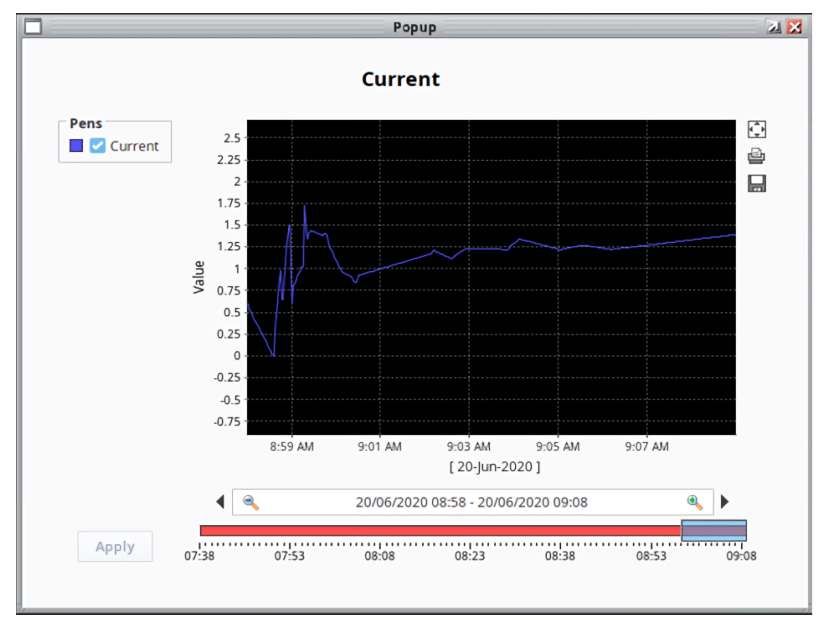

Fig. 17a. Expanded view of recent consumption on the HMI (Current)

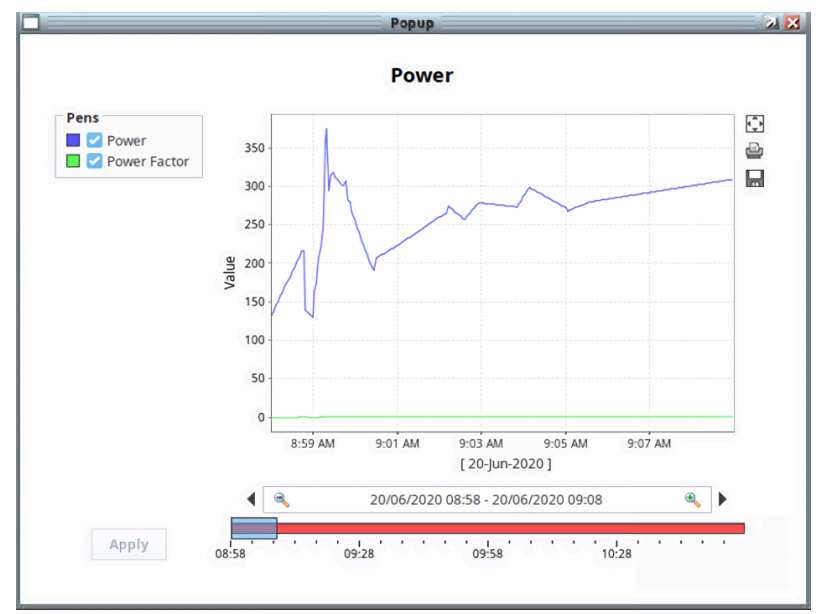

Fig. 17b. Expanded view of recent consumption on the HMI (Power)

sampling speed was selected as $1000 \mathrm{~Hz}$, and the HMI was updated after every 2-3 seconds (which includes the acquisition time and the upload time). Fig. 16 shows the HMI displaying the GPS date-time, RMS Voltage, Current, Power, Power Factor, Frequency, and Relative Amplitude for the harmonics, as well as graphs showing the recent values for voltage, current, and power for when the load was the pedestal fan. It can be observed that the power factor for the fan load is near 0.8 , indicating an inductive load. The HMI also included an overcurrent limit, to disconnect the consumers supply if a certain threshold of electric current was exceeded. This was to act as a preemptive safety feature, as well as a prevention for customers from exceeding a certain usage. The consumer supply could also be remotely switched
OFF, if the need arose, or for the nonpayment of electricity bills and dues resulting in energy theft. This is efficient not only as the remote control feature is convenient and reduces overall costs, but also as the energy cut off this way is faster, and avoids the need for physical confrontation with the customer. Historical plots for current and power are also shown in Fig. 17a and 17b, for a small-time range. The time window could be widened to show older data, and also used to give the load profile for a certain time period.

\section{Acknowledgements}

We would like to express our utmost gratitude to our institution of study, Habib University, which provided us with the percipient knowledge and resources to make completing this paper possible.

\section{Conclusion}

The system that was presented in this paper was able to successfully measure the electricity consumption metrics through the Data Acquisition unit, transmit them to the Gateway through LoRa communication, and then upload them in real-time to the SCADA server, allowing the energy usage to be monitored. Overall, the architecture that was presented was successful in meeting its objectives.

The Proposed System was designed to work according to Pakistans electricity supply specifications, and after calibrations, the voltage measurement on the HMI was able to report a reading with only an error of $+/-1 \mathrm{~V}$ (as was visible in Figure 8. For the current measurement, the demonstration used 5A version of the ACS712 Current Sensor, and its readings had an error of around 3\%, with the error decreasing at higher currents and increasing at lower currents. In actual usage, the current consumption of a household is around 12A to $18 \mathrm{~A}$, so using the $30 \mathrm{~A}$ module would be able to provide lower error percentages in the meter readings. The sampling rate of $1000 \mathrm{~Hz}$ was adequate for accurately sampling the $50 \mathrm{~Hz}$ power frequency. The accuracy of the harmonics and the power factor could not be gauged properly.

Possible enhancements and future development on the project include decreasing inaccuracies in the measurements of the Data Acquisition module, and expanding the system to monitor and analyze multi-phase power lines. Detection of single or multi-phase faults could also be implemented by monitoring the voltage and current regularly, and reporting any unusual changes. The data collected could also be used for analytics and decision making, in the case of scheduling power distribution to certain areas. The voltage and current waveforms could also be analyzed, and AI and Machine Learning could be used to identify the type of loads running at any given time. Consumers could be given the option to notify them if a certain load runs more than a certain amount of time, or if a certain piece of equipment causes unusual electrical transients. Similarly, anti meter tampering features could be introduced to set off alarms in the event of fraud or theft at the meter. 
In terms of the wireless communication, LoRa mesh networks may be used to extend the range of the metering devices, and increased security could be added by encrypting the data being sent over the LoRa communication to prevent hackers from hijacking and modifying the transmissions.

In terms of the HMI, a different consumer facing interface could also be made to allow the customer to access and monitor their usage through an online portal.

The same architecture could also be used for other quantities, such as water or gas flow rate, metering them using the same design with different sensors. A 3-1 Smart Utility Meter, capable of monitoring electricity, gas, and water simultaneously could be created. To combine the principles of energy sharing and net metering, customers could be encouraged to add solar panels or other renewable energy sources to have them linked to the meter, that could provide consumers with saving, and also reduce the load on the grid.

\section{References}

[1] H. Pasha and W. Saleem, "The Impact and Cost of Power Load Shedding to Domestic Consumers," in The Pakistan Development Review, vol. 52, issue 4, Dec 2013. Accessed on: July 2020. [Online]. Available: http://pide.org.pk/psde/pdf/AGM29/papers/Dr.\%20Hafiz\%20P asha.pdf

[2] M. Khan, "Power theft reached over Rs 53 billion in 2017-18," Dawn News, Oct 2018. Accessed on: July 2020. [Online]. Available: https://www.dawn.com/news/1436502

[3] I. Adnan, "Smart Meters: Are they here for good?," MIT Technology Review Pakistan, Oct 2015. Accessed on: July 2020. [Online]. Available: http://www.technologyreview.pk/smart-meters-are-they herefor-good/

[4] A Correspondent, "NEPRA's reply sought on K-Electric's smart meters," The Express Tribune, Oct 2017. Accessed on: July 2020. [Online]. Available: https://tribune.com.pk/story/1541312/1-nepras-reply-soughtkelectrics-smart-meters

[5] G. R. Barai, S. Krishnan, and B. Venkatesh, "Smart metering and functionalities of smart meters in smart grid - a review," 2015 IEEE Electrical Power and Energy Conference (EPEC), 2015, pp. 138-145, doi: 10.1109/EPEC.2015.7379940.

[6] A. S. Metering, S. Visalatchi and K. K. Sandeep, "Smart energy metering and power theft control using Arduino \& GSM," 2017 2nd International Conference for Convergence in Technology (I2CT), 2017, pp. 858-961, doi: 10.1109/I2CT.2017.8226251.

[7] S. V. Sreedevi, P. Prasannan, K. Jiju, and I. J. Indu Lekshmi, "Development of Indigenous Smart Energy Meter adhering Indian Standards for Smart Grid," 2020 IEEE International Conference on Power Electronics, Smart Grid and Renewable Energy (PESGRE2020), 2020, pp. 1-5, doi: 10.1109/PESGRE45664.2020.9070245.

[8] A. R. Al-Ali, T. Landolsi, M. H. Hassan, M. Ezzeddine, M. Abdelsalam and M. Baseet, "An IoT-Based Smart Utility Meter," 2018 2nd International Conference on Smart Grid and Smart Cities (ICSGSC), 2018, pp. 80-83, doi: 10.1109/ICSGSC.2018.8541314.

[9] H. Shahinzadeh, J. Moradi, G. B. Gharehpetian, H. Nafisi and M. Abedi, "IoT Architecture for Smart Grids," 2019 International Conference on Protection and Automation of Power System (IPAPS), 2019, pp. 22-30, doi: 10.1109/IPAPS.2019.8641944.

[10] M. C. Falvo, L. Martirano, D. Sbordone, and E. Bocci, "Technologies for smart grids: A brief review," 2013 12th International Conference on Environment and Electrical Engineering, 2013, pp. 369-375, doi: 10.1109/EEEIC.2013.6549544.

[11] Z. Davoody-Beni, N. Sheini-Shahvand, H. Shahinzadeh, M. Moazzami, M. Shaneh and G. B. Gharehpetian, "Application of IoT in Smart Grid: Challenges and Solutions," 2019 5th Iranian Conference on Signal Processing and Intelligent Systems (ICSPIS), 2019, pp. 1-8, doi: 10.1109/ICSPIS48872.2019.9066025

[12] A. Shobol, M. H. Ali, M. Wadi, and M. R. TüR, "Overview of Big Data in Smart Grid," 2019 8th International Conference on Renewable Energy Research and Applications (ICRERA), 2019, pp. 1022-1025, doi: 10.1109/ICRERA47325.2019.8996527.

[13] G. Wibisono, S. G. Permata, A. Awaludin, and P. Suhasfan, "Development of advanced metering infrastructure based on LoRa WAN in PLN Bali toward Bali Eco smart grid," 2017 Saudi Arabia Smart Grid (SASG), 2017, pp. 1-4, doi: 10.1109/SASG.2017.8356496 